\title{
Cancer drugs: remedy required
}

\author{
Cancer drugs often impair quality of life and fail to extend patient survival. Mandating increased efficacy and \\ promoting efforts to target tumor metastasis may improve outcomes for patients with cancer.
}

A recent report by researchers at the US National Cancer Institute (NCI) estimates that cancer costs in the US for 2010 exceeded $\$ 124$ billion and may top $\$ 157$ billion (in 2010 dollars) by 2020, owing to population growth and aging (J. Natl. Cancer Inst. 103, 117-128, 2011). The authors concluded that, on a per-person basis, the cost of cancer is highest in the last year of life and will likely increase further as new technologies and new targeted therapies enter the clinic.

But, all too often, new therapies extend patients' lives by just days to months, and the quality of life added is questionable at best. Can cancer care dollars be better spent? In another article, Ocana and Tannock suggest that one way to improve returns on cancer spending is for regulatory agencies to mandate stronger evidence of efficacy for the approval of new cancer drugs (J. Natl. Cancer Inst. 103, 16-20, 2011).

The authors analyzed the results of 18 clinical trials used for the approval of ten cancer drugs by the US Food and Drug Administration (FDA) between 2000 and 2010. At the start of a trial, investigators specify the difference they wish to detect in the primary endpoint-usually overall survival or progression-free survival-in patients treated with the investigational new drug compared with the standard of care. The magnitude of this difference is not chosen to reflect a clinically meaningful benefit and is instead selected on the basis of what can be detected within the particular sample size of a trial-in other words, the larger the number of trial participants, the smaller the difference can be while remaining statistically significant. For instance, in a phase 3 trial of erlotinib for the treatment of pancreatic cancer, an increase in median overall survival of only ten days was statistically significant. Moreover, if patient enrollment is reopened, as occurred in another of the trials reviewed, the resultant larger trial increases the likelihood of observing a statistically significant difference between the treatment arms.

But statistically significant is not synonymous with clinically important, and Ocana and Tannock advocate the use of strict measures to define a positive clinical cancer trial. They suggest that approval of a cancer drug should require evidence of a minimum 3-month extension to overall survival or a 4-6 month increase in progression-free survival. Such stringent criteria would have precluded the approval of a number of cancer drugs currently in clinical use.

But is a drug that extends survival by a few days, and may compromise quality of life due to adverse side effects, of clinical value to patients? The use of bevacizumab (Avastin; Genentech/Roche) for the treatment of metastatic breast cancer is a case in point. The FDA approved bevacizumab for the treatment of chemotherapy-naive metastatic breast cancer in combination with paclitaxel in 2008 , based on a phase III trial showing a median increase in progression-free survival of 5.9 months. But trials completed after its approval showed a substantially smaller increase in progression-free survival, no increase in overall survival and life-threatening complications. As a result, the FDA revoked its recommendation of bevacizumab for the treatment of breast cancer in December 2010, indicating that the drug has not proved safe enough or of sufficient clinical benefit to this patient population to outweigh its risks.

Certainly not all patients agree with this decision, and pharmaceutical companies may be loath to test new cancer drugs if more stringent regulatory requirements increase the likelihood of failure and therefore of research and development costs. But by mandating clinically important benefits and a more rigorous examination of candidate drugs, regulatory agencies may help reallocate resources to more directly target the processes mediating tumor progression and ultimately tumor metastasis.

Because although tumor metastasis accounts for $90 \%$ of cancerrelated deaths, most existing cancer therapies were not developed to specifically block the metastatic process. Nor do pharmaceutical companies or academia invest heavily in metastasis research: just $7.7 \%$ of NCI-funded research in 2009 was classified as metastasis and European funding agencies support metastasis research at similarly low rates (Euro. J. Cancer 46, 1177-1180, 2010).

This comparative lack of resources to study metastasis is no doubt partly due to its complexity. Whereas studies of oncogenic viruses, tumor cell lines and primary tumors in humans and in animal models have provided a wealth of information on the mechanisms of cell transformation and tumor cell growth, the more dynamic, and possibly stochastic, process of metastasis has been far more difficult to model. The mechanisms responsible for the ability of a small fraction of tumor cells to invade the extracellular matrix, intravasate blood and lymphatic vessels, survive in the circulation, extravasate and colonize a distant tissue or, conversely, go dormant are, to a large degree, unknown. Characteristics that clearly distinguish metastases from primary tumors are difficult to define, and the intrinsic roles of the tissue microenvironment and of inherited susceptibility in modulating the metastatic process remain to be elucidated. These biological questions, coupled with the practical problems of targeting disseminated tumor cells, monitoring at a molecular level drug efficacy in metastases and elucidating the drug sensitivity of such cells relative to the bulk tumor, in addition to the scarcity of biopsy samples of metastases as source material to help answer some of these questions, all contribute to the difficulties in specifically studying and targeting tumor metastasis.

As a result, we presently have a collection of drugs that are not selective for the inhibition of metastasis and whose determination of efficacy is based on whether they confer a statistically significant-but not necessarily clinically meaningful-increase in overall survival or progression-free survival. Encouraging investment in inhibitors of metastasis, coupled with the development of therapies that achieve discrete measures of efficacy, may help truly attain the goal of longer survival for patients with cancer.

Corrected after print 11 March 2011. 


\section{ER R ATA}

\section{Erratum: Cancer drugs: remedy required}

\section{Nat. Med. 17, 231 (2011); published online 7 March 2011; corrected after print 11 March 2011}

In the version of this article initially published, the manufacturer of bevacizumab (Avastin) was reported incorrectly as Merck. The manufacturer of bevacizumab is Genentech/Roche. The error has been corrected in the HTML and PDF versions of the article.

The article also states that the US Food and Drug Administration (FDA) approved bevacizumab on the basis of a phase 3 trial that showed a median increase in progression-free survival of 5.9 months, which is consistent with the published literature (N. Engl. J. Med. 357, 2666-2676, 2007). An independent review of the data concluded that the progression-free survival difference was 5.5 months, which is listed on the FDA-approved label for bevacizumab.

On December 15, the FDA's Office of New Drugs (OND) issued a decision memo on Avastin, which states that "it is the conclusion of OND that the breast cancer indication for Avastin be withdrawn." The FDA has granted Genentech/Roche a public hearing on the matter, to be held in June 2011. 